\title{
Decay of Cosmic String Loops due to Particle Radiation
}

\author{
Daiju Matsunami, ${ }^{1,2}$ Levon Pogosian, ${ }^{2}$ Ayush Saurabh, ${ }^{3}$ and Tanmay Vachaspati ${ }^{3}$ \\ ${ }^{1}$ Canadian Institute for Theoretical Astrophysics, University of Toronto, Toronto, Ontario M5S 3H8, Canada \\ ${ }^{2}$ Physics Department, Simon Fraser University, Burnaby, British Columbia V5A 1S6, Canada \\ ${ }^{3}$ Physics Department, Arizona State University, Tempe, Arizona 85287, USA
}

(Received 12 March 2019; published 20 May 2019)

\begin{abstract}
Constraints on the tension and the abundance of cosmic strings depend crucially on the rate at which they decay into particles and gravitational radiation. We study the decay of cosmic string loops in the AbelianHiggs model by performing field theory simulations of loop formation and evolution. We find that our set of string loops emits particle radiation primarily due to kink collisions, and that the decay time due to these losses is proportional to $L^{p}$ with $p \approx 2$ where $L$ is the loop length. In contrast, the decay time to gravitational radiation scales in proportion to $L$, and we conclude that particle emission is the primary energy loss mechanism for loops smaller than a critical length scale, while gravitational losses dominate for larger loops.
\end{abstract}

DOI: 10.1103/PhysRevLett.122.201301

Cosmic strings play an important role in building theories of the early Universe [1] and provide a rare observational probe of string theory [2]. The search for their signatures has mostly focused on their gravitational effects, and they are among the main science goals of LIGO [3]. The tightest bound on the string tension $\mu$, coming from millisecond pulsar timing measurements [4], is based on the gravitational wave (GW) background produced by decaying cosmic string loops. This bound, $G \mu \lesssim 10^{-10}$ [3,5], where $G$ is Newton's gravitational constant, assumes that string loops decay primarily into $\mathrm{GW}$ with the quantitative predictions obtained from simulations using the Nambu-Goto (NG) approximation that ignores the field composition of the strings [6-10]. While it is widely accepted that the NG description works well for loops much larger than the string width, the exact loop size above which the particle composition of the string cores can be ignored is not firmly established. The few existing field theory simulations of string networks suggest that loops primarily decay into particle radiation [11], with cosmological size loops not surviving beyond one oscillation, potentially leading to a new paradigm for cosmic string evolution in which the GW bounds do not apply. Thus, it is critical to examine particle emission by cosmic string loops and to determine their primary decay mode.

Previous studies of the particle radiation from cosmic strings included analytical estimates [12], some based on

Published by the American Physical Society under the terms of the Creative Commons Attribution 4.0 International license. Further distribution of this work must maintain attribution to the author(s) and the published article's title, journal citation, and DOI. Funded by SCOAP ${ }^{3}$. effective couplings of NG strings to other fields [13,14], field theory simulations of standing waves, kinks and cusps on long strings [15,16], and simulations of strings with small oscillations $[11,17]$. In this work, for the first time, we directly examine the decay of a cosmic string loop to particle radiation in the Abelian-Higgs model by simulating loop formation followed by evolution in full field theory. The focus on a single loop is to be contrasted with the very large field theory simulations of an entire network of strings in an expanding spacetime $[11,18]$.

We find that string loops emit particle radiation mainly due to features on the strings known as kinks and cusps [1]. The half-life of a loop due to particle radiation is proportional to $L^{p}$, where $L$ is the length of the loop and $p \approx 2$ for the loops we have considered. On the other hand, the loop half-life due to gravitational radiation is known to be proportional to $L$. Thus, there is a crossover from particle decay to gravitational decay roughly given by $L_{*} \sim w / G \mu$ where $w \sim \mu^{-1 / 2}$ is the width of the string. For $L<L_{*}$, loops decay by particle emission, while for $L>L_{*}$ gravitational emission dominates. We discuss caveats and the implications of this result in more detail below, along with the values of $p$ that might arise for loops other than those we have directly simulated.

We consider the Abelian-Higgs field theory with a complex scalar field, $\phi=\phi_{1}+i \phi_{2}$, and a U(1) gauge field $A_{\mu}$. We work in the temporal gauge, $A_{0}=0$, and the field equations of motion are

$$
\begin{aligned}
\partial_{t}^{2} \phi_{a}= & \nabla^{2} \phi_{a}-e^{2} A_{i} A_{i} \phi_{a}-2 e \epsilon_{a b} \partial_{i} \phi_{b} A_{i}-e \epsilon_{a b} \phi_{b} \Gamma \\
& -\lambda\left(\phi_{b} \phi_{b}-\eta^{2}\right) \phi_{a}, \\
\partial_{t} F_{0 i} & =\nabla^{2} A_{i}-\partial_{i} \Gamma+e\left(\epsilon_{a b} \phi_{a} \partial_{i} \phi_{b}+e A_{i} \phi_{a} \phi_{a}\right),
\end{aligned}
$$




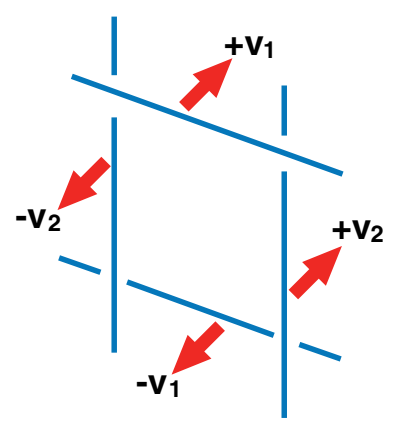

FIG. 1. Schematic representation of the initial configuration. Four straight strings are set up with velocities as shown. The strings intersect and reconnect to produce a central loop and also a second "outer" loop because of periodic boundary conditions. These loops then oscillate and shrink without interacting with each other. By choosing the spacing of the initial strings, we can produce loops of different sizes.

$$
\partial_{t} \Gamma=\partial_{i} F_{0 i}-g_{p}^{2}\left[\partial_{i} F_{0 i}+e \epsilon_{a b} \phi_{a} \partial_{t} \phi_{b}\right],
$$

where $a=1,2, \epsilon_{a b}$ is the Levi-Civita tensor with $\epsilon_{12}=1$, $F_{0 i}=\partial_{t} A_{i}$ in the temporal gauge, $\lambda$ and $e$ are coupling constants, $\Gamma \equiv \partial_{i} A_{i}$, and $g_{p}^{2}$ is a parameter introduced for numerical stability [19]. The solution for a topologically stable straight string along the $z$ axis is [20]

$$
\phi=\eta f(r) e^{i \theta}, \quad A_{i}=v(r) \epsilon_{i j} \frac{x^{j}}{r^{2}} \quad(i, j=1,2),
$$

where $r=\sqrt{x^{2}+y^{2}}, \theta=\tan ^{-1}(y / x)$, and $f(r)$ and $v(r)$ are profile functions that vanish at the origin and asymptote to 1 , respectively. The string energy per unit length (also its tension) is given by $\mu=\pi \eta^{2} F(\beta)$ where $\beta \equiv 2 \lambda / e^{2}$ and $F$ is a numerically determined function such that $F(1)=1$. We will only consider $\beta=1$ corresponding to the Bogomol'nyi-Prasad-Sommerfield (BPS) limit [21,22], where $\mu=\pi \eta^{2}$ and the scalar mass $m_{S}=\sqrt{2 \lambda} \eta$ equals the vector mass $m_{V}=e \eta$.

Our aim is to produce a loop as might be produced in a cosmological setting and then to evolve it. For this purpose, we set up initial conditions with four straight strings that are moving with velocities $\pm \mathbf{v}_{\mathbf{1}}$ and $\pm \mathbf{v}_{\mathbf{2}}$ as shown schematically in Fig. 1. The four strings then collide to form a loop with a stationary center of mass and a nonzero angular momentum. The latter is essential to prevent the loop from simply collapsing to a double line. Preparing this initial configuration starts with taking the string solution of Eq. (4) oriented along a given direction, boosting it to a suitable velocity, and gauge transforming the boosted solution back in to the temporal gauge. Then the four string solutions have to be patched together in a simulation box with periodic boundaries. Further details are provided in the Supplemental Material [23].

Cosmological strings are expected to be mildly relativistic and we choose $\left|\mathbf{v}_{1}\right|=0.6$ and $\left|\mathbf{v}_{2}\right|=0.33$. The directions are taken to be $\left(\hat{v}_{1}\right)_{x}=0.4, \quad\left(\hat{v}_{1}\right)_{y}=$ $\sqrt{1-0.4^{2}} \approx 0.92$ for the two strings oriented along the $z$ axis and $\left(\hat{v}_{2}\right)_{z}=0.4,\left(\hat{v}_{2}\right)_{y} \approx 0.92$ for those along the $x$ axis. The string velocities are approximately aligned along the $y$ axis, but not exactly, to avoid overly symmetrical loops that tend to pass through a double line configuration and collapse prematurely. We have experimented with a wide range of initial velocities and our main conclusions are independent of the particular choices of these parameters.

Given the initial conditions for fields $\phi, A_{\mu}$, we evolved them using the discretized version of Eqs. (1)-(3) with $e=1, \lambda=1 / 2, \eta=1$, and $g_{p}^{2}=0.75$. We used the explicit Crank-Nicholson algorithm with two iterations for the evolution [24] and periodic boundary conditions. We tried different lattice spacings to study the effects of numerical resolution. The initial string spacing was set to a fixed fraction of the simulation box size so that smaller loops ran in a smaller box, with less computational cost.

Because of periodic boundary conditions, the reconnection of four strings produces two loops - the central loop in the middle of the box shown in Fig. 1, and an outer loop formed from the "fragments" in the corners of the box. The two loops then oscillate and decay without intersecting each other. We track the loop energy by summing the energy density in the "core" of the string. The energy density is given by

$$
\mathcal{E}=\frac{1}{2}\left|D_{0} \phi\right|^{2}+\frac{1}{2}\left|D_{i} \phi\right|^{2}+\frac{1}{2}\left(\mathbf{E}^{2}+\mathbf{B}^{2}\right)+\frac{\lambda}{4}\left(|\phi|^{2}-\eta^{2}\right)^{2},
$$

where $\mathbf{E}$ and $\mathbf{B}$ are the electric and magnetic field vectors, with their components defined as $E_{i}=F_{0 i}$ and $B_{i}=-\frac{1}{2} \epsilon_{i j k} F_{j k}$. We define the string core to be the cells where the magnitude of the scalar field $|\phi|$ is less than $0.9 \eta$.

In Fig. 2 we plot the loop energy vs time for a simulation on a $600^{3}$ lattice with $\Delta x=0.25$, where the initial size of

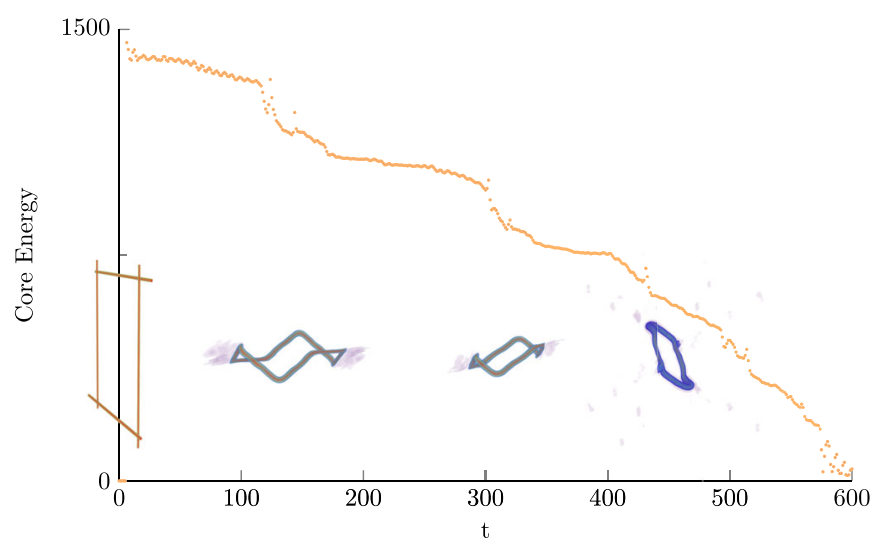

FIG. 2. Energy of a loop with the initial size of 390 lattice spacings plotted vs time. Overlaid on the plot are snapshots of the loop as it goes through phases of rapid radiation discharge due to smoothening of kinks. The animation showing the evolution of this loop can be found at [25]. 
TABLE I. Loop sizes in lattice units for each of the runs.

\begin{tabular}{lcc}
\hline \hline Lattice size & Inner loop & Outer loop \\
\hline $400^{3}$ & 140 & 260 \\
$600^{3}$ & 210 & 390 \\
$800^{3}$ & 280 & 520 \\
$1200^{3}$ & 420 & 780 \\
\hline \hline
\end{tabular}

the loop is 390 lattice spacings. (The animation of the loop evolution can be found at [25].) The plot suggests episodic radiation, with the overlaid snapshots showing the representative "events" leading to drops in the loop energy. Straight strings do not radiate as they correspond to a boosted string solution. The kinks on the loop, formed at the intercommutation of the straight strings, also propagate with minimal energy loss. We find that noticeable radiation is produced when kinks collide. Also, as the kinks smooth out, there are episodes of large radiation which may be due to the formation of weak cusps. Particle radiation from cusps was studied in Ref. [15] where it was found that the energy emission from a cusp leads to the formation of kinks and to weak cusps in subsequent loop oscillations. This pattern of episodic radiation from kink collisions and weak cusps, with relatively minor energy loss in between these events, is common to all loop simulations we have performed.

To obtain a quantitative measure of the scaling of the loop half-life with its size, we have run simulations for four different box sizes yielding eight loops given in Table I. (Two loops from different runs are almost the same length and provide a check on our simulation.) Figure 3 shows the loop energy versus time for the eight loops. As the loops evolve, they also shed their angular momentum, defined as

$$
\begin{aligned}
L_{i} \equiv & \epsilon_{i j k} \int_{\text {string core }} d^{3} x x_{j}\left(-\frac{1}{2}\left[\left(D_{0} \phi\right)\left(D_{k} \phi\right)^{*}\right.\right. \\
& \left.\left.+\left(D_{0} \phi\right)^{*}\left(D_{k} \phi\right)\right]+\epsilon_{k l m} E_{l} B_{m}\right) .
\end{aligned}
$$

In Fig. 4 we plot $|\mathbf{L}|$ vs time and also see episodic decay. We have run our simulations for a few different values of the lattice spacing $\Delta x$ and found that the results are sensitive to the resolution. For example, as shown in Fig. 5, the total energy in the simulation box over the entire run is conserved only at the $\sim 33 \%$ level when $\Delta x=0.50$. For $\eta=1, e=1, \lambda=\frac{1}{2}$, the string width is $\sim 1$. Therefore, with $\Delta x=0.5$ we have only a few lattice points within the width of the string. Using $\Delta x=0.25$ improves the conservation to the $\sim 5 \%$ level and agrees well with the much more computationally expensive run with $\Delta x=0.125$. The choice of $\Delta x$ makes an important difference in the lifetime of the loop, as is clear from the right panel of Fig. 5. Loops live longer in simulations with better resolution. From the animations, we see that the shorter

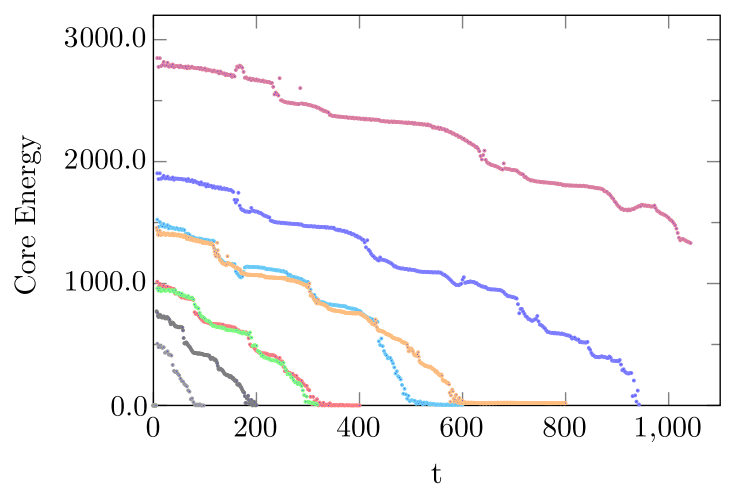

FIG. 3. Loop energy vs time for eight different loops in four separate runs at $\Delta x=0.25$ resolution.

loops live for about one oscillation period while the larger loops survive for several oscillation periods. (There is ambiguity in defining an oscillation period since the length of the loop and hence its oscillation period is changing relatively rapidly during the simulation.)

The longest loop we are able to simulate has an energy of $\sim 3 \times 10^{3}$, which corresponds to length $L \sim 10^{3} \mathrm{w}$ where $\mathrm{w}$ is the width of the string. In cosmology we are interested in loops of length comparable to the cosmic horizon, which is orders of magnitude larger than the thickness of the string, perhaps by a factor of $\sim 10^{60}$. So we need to extrapolate our results to larger lengths. For this purpose we calculate the half-life $\tau$, i.e., the time it takes the loop to lose half its initial energy. In Fig. 6 we plot $\tau / \tau_{0}$, where $\tau_{0}=41.5 / \eta$ is the half-life of the smallest loop in our simulations, versus the initial energy normalized by that of the smallest loop (denoted $E_{0}=506 \eta$ ). We find a power law fit

$$
\tau=\tau_{0}\left(\frac{E}{E_{0}}\right)^{p}=\frac{1.6 \times 10^{-3}}{\eta}(\eta L)^{p}, \quad p \approx 2,
$$

where we have reinserted dimensional factors of $\eta$.

The $L^{2}$ scaling in (7) can be understood as following from radiation being due to episodes involving a fixed number of features (kinks and weak cusps) on the loop,

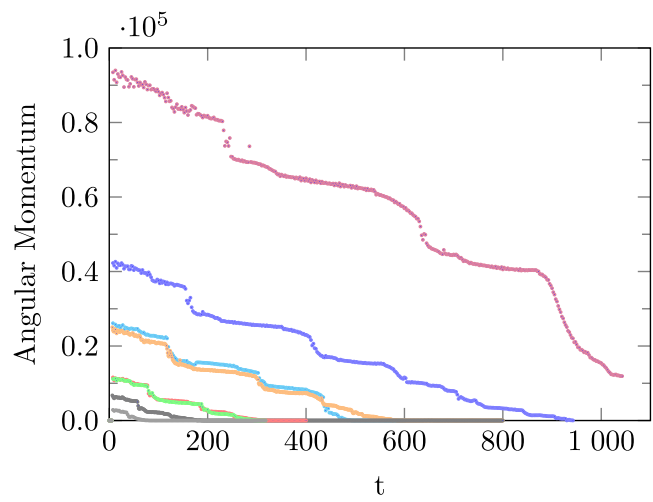

FIG. 4. Loop angular momentum vs time for eight different loops in four separate runs at $\Delta x=0.25$ resolution. 

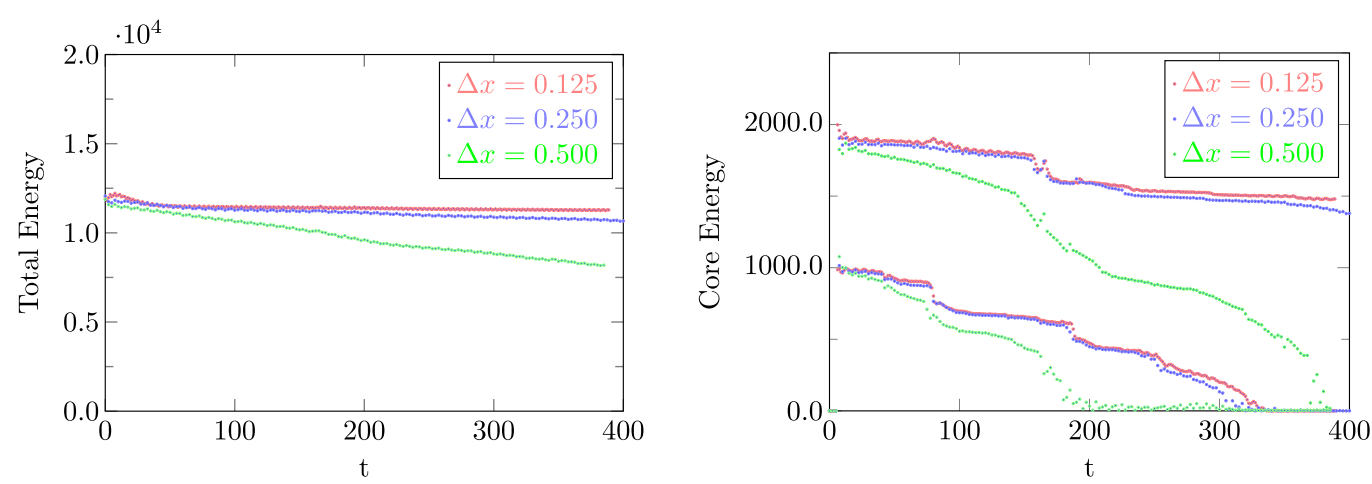

FIG. 5. Comparison of runs with different lattice resolution $\Delta x=0.125,0.25,0.5$ on lattices of size 1600 , 800, and 400, respectively, corresponding to a fixed physical lattice length of 100. The left panel shows the total energy in our simulation box and the right panel shows the evolution of the energy in the two loops in the box. The plots show convergence at higher resolution and that $\Delta x=0.25$ offers a good compromise between accuracy and speed.

with the power emitted in a given episode (a kink collision or a weak cusp) being independent of $L$. (Note that the size of the steps seen in Fig. 3 is similar for different loops.) If $\nu$ denotes the number of episodes per period and each episode radiates energy $\epsilon$ on average, the energy lost per unit time is

$$
\dot{E} \sim-\frac{\nu \epsilon}{L} \sim-\frac{\mu \nu \epsilon}{E}
$$

Integration of this equation gives a lifetime

$$
\tau \sim \frac{E^{2}}{\mu \nu \epsilon} \sim \frac{\mu L^{2}}{\nu \epsilon}
$$

in agreement with the $L^{2}$ scaling in (7).

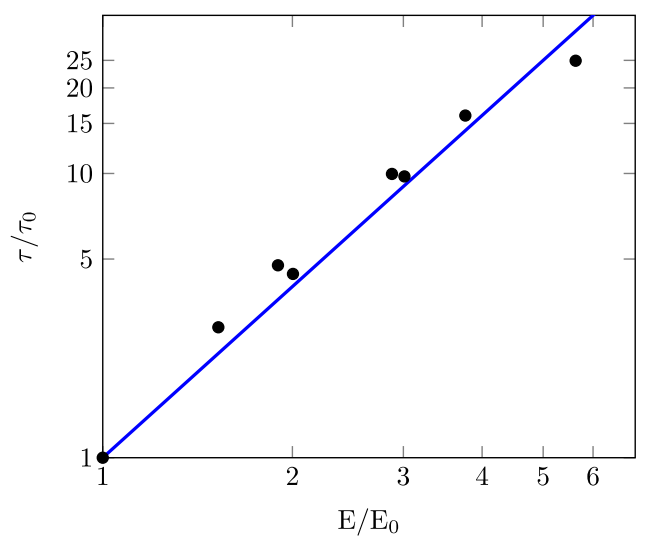

FIG. 6. Plot of the loop half-life versus initial energy (proportional to the initial length) in the loop. They are normalized by the initial half-life of the smallest loop $\tau_{0}$ and its energy $E_{0}$. The straight line fit shows that $\tau \propto L^{2}$ where $L$ is the initial length of the loop.
The particle radiation rate (8) is to be contrasted with $\dot{E} \sim \nu G \mu^{2}$ expected due to gravitational wave radiation from $\nu$ radiation episodes involving kinks and cusps [26-28]. Note that the rate of energy loss to gravitational radiation is not suppressed by a factor of $L$ as is the case for particle radiation in (8). This is because, e.g., a cusp on a loop that is twice as large is also twice as large, and the gravitational energy emitted by a single cusp is proportional to $L$. Then the lifetime of the loop due to gravitational radiation is

$$
\tau_{g} \sim \frac{L}{\nu G \mu}
$$

Comparing this to (9) allows us to derive a criterion for when the gravitational radiation is more important than particle radiation, namely, when

$$
\tau_{g}<\tau \Rightarrow L \gtrsim \frac{\epsilon}{G \mu^{2}} \sim \frac{w}{G \mu},
$$

where $w$ is the string thickness and we estimate $\epsilon \sim \sqrt{\mu}$; i.e., the particle energy emitted in an episode is comparable to the energy scale of the string, and $l_{P} \sim 10^{-33} \mathrm{~cm}$ is the Planck length. Note that $\nu$ has canceled out in (11). Therefore, even if there are more episodes on larger loops, gravitational radiation still dominates over particle radiation if (11) is satisfied.

With $L \sim 10^{27} \mathrm{~cm}$ we find that gravitational radiation is less important than particle radiation if $G \mu \lesssim 10^{-40}$, corresponding to $\eta \sim 100 \mathrm{MeV}$ or the QCD scale. Hence, particle radiation could be the main decay mechanism for strings formed below the QCD scale but the dynamics of strings formed at such low energies is expected to be dominated by friction with the ambient medium [1].

Alternately, for strings close to the current bound on the string tension, $G \mu \approx 10^{-11}$, Eq. (11) implies that particle radiation will only be important for loops that are very 
small, $L<10^{-17} \mathrm{~cm}$. Most of the radiation from such a network of strings will be in gravitational waves.

We would like to point out some caveats to the above discussion. The first caveat is that the long strings in our initial conditions are straight and smooth. If these strings started out with structure (perhaps as shallow kinks) on them, as has been suggested in Ref. [18], the number of radiative episodes would be larger, and both the particle and gravitational radiation would be larger. This would not change the relative importance of particle and gravitational radiation but it would mean that the loop decays faster. A second caveat is that our loops only contain kinks and no cusps. It is known from Ref. [15] that the particle radiation loss from a cusp is proportional to $\sqrt{L}$ and this does not agree with our model where each episode emits radiation that is independent of $L$. However, once the cusp radiates, it forms two kinks that then propagate, radiate, and smooth out to some extent. In the next oscillation, the cusp is weaker and the energy radiated will not be proportional to $\sqrt{L}$, instead it will be proportional to some power of $L$ smaller than $1 / 2$. Thus, with cusps we expect that the effective value of $p$ will satisfy $1<p<3 / 2$, and Eq. (11) will get modified. Even then there will be a critical loop length such that gravitational emission dominates over particle radiation for larger loops. A third caveat is that since our initial strings were straight, there was no radiation while the kinks propagate on the straight segments. If, however, the segments are curved, there will be some radiation even from a propagating kink. This radiation would not be episodic but it would be suppressed by the curvature of the segment, expected to be suppressed by the loop size divided by the cosmic horizon scale.

To summarize, we have studied the formation and evolution of cosmic string loops in field theory and estimated their lifetimes. We find that the lifetime of the loops is very sensitive to the resolution used in their numerical evolution. With insufficient resolution, the loops collapse within one oscillation period. At higher resolution, the loops survive for a few oscillation periods and we observe that their lifetime grows as $L^{2}$. We can explain this growth in terms of episodic particle radiation. When compared to gravitational energy losses, we find that gravitational radiation dominates for loops that are larger than a critical length [see Eq. (11)].

We thank the Lorentz Center for hosting the Topological Defects workshop where we had the opportunity to discuss these results with the participants, especially Jose BlancoPillado, Mark Hindmarsh, Ken Olum, Paul Shellard, and Daniele Steer. D. M. and L. P. are supported in part by the National Sciences and Engineering Research Council (NSERC) of Canada. A.S. and T. V. are supported by the U.S. Department of Energy, Office of High Energy Physics, under Award No. DE-SC0019470 at Arizona State University. This research was enabled in part by support provided by WestGrid [29] and Compute Canada [30]. The bulk of the computations were performed on the Agave and Stampede2 clusters at Arizona State University and The University of Texas at Austin, respectively.

[1] A. Vilenkin and E.P.S. Shellard, Cosmic Strings and Other Topological Defects (Cambridge University Press, Cambridge, England, 2000), http://www.cambridge .org/mw/academic/subjects/physics/theoretical-physics-andmathematical-physics/cosmic-strings-and-other-topologicaldefects?format=PB.

[2] E. J. Copeland, L. Pogosian, and T. Vachaspati, Classical Quantum Gravity 28, 204009 (2011).

[3] B. Abbott et al. (LIGO Scientific and Virgo Collaborations), Phys. Rev. D 97, 102002 (2018).

[4] P. D. Lasky et al., Phys. Rev. X 6, 011035 (2016).

[5] J. J. Blanco-Pillado, K. D. Olum, and X. Siemens, Phys. Lett. B 778, 392 (2018).

[6] A. Albrecht and N. Turok, Phys. Rev. Lett. 54, 1868 (1985).

[7] D. P. Bennett and F. R. Bouchet, Phys. Rev. D 41, 2408 (1990).

[8] B. Allen and E. P. S. Shellard, Phys. Rev. Lett. 64, 119 (1990).

[9] J. J. Blanco-Pillado, K. D. Olum, and B. Shlaer, Phys. Rev. D 83, 083514 (2011).

[10] L. Lorenz, C. Ringeval, and M. Sakellariadou, J. Cosmol. Astropart. Phys. 10 (2010) 003.

[11] M. Hindmarsh, J. Lizarraga, J. Urrestilla, D. Daverio, and M. Kunz, Phys. Rev. D 96, 023525 (2017).

[12] T. Vachaspati, A. E. Everett, and A. Vilenkin, Phys. Rev. D 30, 2046 (1984).

[13] M. Srednicki and S. Theisen, Phys. Lett. B 189, 397 (1987).

[14] R. H. Brandenberger, Nucl. Phys. B293, 812 (1987).

[15] K. D. Olum and J. J. Blanco-Pillado, Phys. Rev. D 60, 023503 (1999).

[16] K. D. Olum and J. J. Blanco-Pillado, Phys. Rev. Lett. 84, 4288 (2000).

[17] C. J. A. P. Martins, J. N. Moore, and E. P. S. Shellard, Phys. Rev. Lett. 92, 251601 (2004).

[18] M. Hindmarsh, S. Stuckey, and N. Bevis, Phys. Rev. D 79, 123504 (2009).

[19] T. Vachaspati, Phys. Rev. Lett. 117, 181601 (2016).

[20] H. B. Nielsen and P. Olesen, Nucl. Phys. B61, 45 (1973).

[21] E. B. Bogomolny, Yad. Fiz. 24, 861 (1976) [Sov. J. Nucl. Phys. 24, 449 (1976)].

[22] M. K. Prasad and C. M. Sommerfield, Phys. Rev. Lett. 35, 760 (1975).

[23] See Supplemental Material at http://link.aps.org/ supplemental/10.1103/PhysRevLett.122.201301 for details involved in setting up the initial field configuration of four strings in our simulation.

[24] S. A. Teukolsky, Phys. Rev. D 61, 087501 (2000).

[25] https://ayushsaurabh.home.blog.

[26] T. Vachaspati and A. Vilenkin, Phys. Rev. D 31, 3052 (1985).

[27] D. Garfinkle and T. Vachaspati, Phys. Rev. D 36, 2229 (1987).

[28] P. Binetruy, A. Bohe, T. Hertog, and D. A. Steer, Phys. Rev. D 80, 123510 (2009).

[29] http://www.westgrid.ca.

[30] http://www.computecanada.ca. 